\title{
Modélisation numérique du comportement d'un sol gonflant chargé soumis à des variations hydriques
}

M. MRAD, A. ABDALLAH, F. MASROURI

Laboratoire Environnement Géomécanique et Ouvrages Nancy-Unversité Rue du Doyen Marcel Roubault $B P 40$

54501 Vandceuvre-lèsNancy Cedex

Moharnad.Mrad@ensg.inplnancy.tr

Adel.Abdallah@ensg.inplnancy.fr

Farimah.Masrouri@ensg. inpl-nancy. $f$
Les phénomènes de retrait-gonflement des sols argileux se manjfestent par des désordres affectant principalement des maisons individuelles souvent peu rigides et fondées superficiellement. En effet, l'alternance des périodes de sécheresse et de précipitation entraîne des modifications de l'état hydrique du sol se traduisant par des déplacements des fondations superficielles. Cette alternance de retrait et de gonflement peut provoquer des dègāts dants les bâtís sous forme de fissures, voire entraîner la rupture partielle ou totale de l'ouvrage, lorsqu'ils ne sont pas pris en compte dans les projets. Dans cet artícle, le modẻle élastoplastique BExM (Barcelona Expansive Model), pour les sols gonflants non saturés implanté dans le code de calcul aux éléments finis Code_Bright, est utilisé pour l'étude du comportement hydromécanique d'un sol gonflant chargé par une semelle filante. L'objectif de cette étude est d'analyser l'influence des sollicitations hydriques dues à la variation des conditions climatiques (précipitation et évaporation) sur les déplacements du massif de sol argileux, et d'étudier I'influence d'une géomembrane posée dans le sol afin de retarder les transferts hydriques et les phénomènes de retrait-gonflement. Les résultats obtenus montrent que le modèle est capabie de prédire qualitativement les déplacements du massif de sol pendant les différentes sollicitations hydriques.

Mots ‘clés : sol gonflant non saturé, couplage hydromécanique, modélisation numérique, éléments finis. fondation superficielle, géomembrane, modèle BExM.

One of the primary causes of shallow foundawon problems in many regions is the highly expansive nature of the clayey soil on which the buildings rest. The clay expands of contracts as its moisture content changes with the variation of the climatic conditions. Indeed, the altemation of rainfall and drought periods irvolves modifications of the soil hydric state resulting in displacements. Wher not taken into account in the project design this altemation of shrinkage and swelling can damage buildings by inducing cracks and partial or total failure of the structure. In this paper, the elastoplastic model BExM (Barcelona Expansive Model) for swelling unsaturated soils implemented in the finite-element program Code_Bright is used for the study of the hydromechanical behaviour of a swelling soil loaded by a strip foundation. The objective of this study is to analyze the effects of hydric solicitations due to variations of the climatic conditions (precipitation and evaporation) on the displacements of the clayey soil and to study the effect of a geomembrane protection posed in the soli an order to delay the hydraulic transfer and the shrinkage-sweling of the soil. 
The obtained results show that the numerical model is able to qualitatively predict the soil displacements during various hydric solicititions.

Key words: urssaturated swelling soil; hydromechanical coupling; Finite-Element method; shallow foundation; geomembrane; BExM model.

\section{Introduction}

Certains sols argileux présentent des variations de volume importantes liées à la variation de leur teneur en eau en lonction des conditions climatiques. Ces variations de volume sont à l'origine de nombreux désordres tant pour les constructions en surface (fondations superficielles, ouvrages de soutènement, remblais, barrages en terre....) notamment quand elles provoguent des déformations différentielles, que pour les ouvrages enterrés (tunnels, canalisations, fondations profondes...). Les exemples de désordres liés à la présence d'argiles gonflantes sont nombreux et variés (Chen, 1975 ; Philipponat, 1991 ; Vandangeon, 1992 ; Derriche et al, 1999; Hachichl et Fleureau, 1999 ; etc.). En France, depuis les sécheresses des années 19891991, 1997-1998 et 2003, qui ont affecté notamment des constructions fondées superficiellement, les conséquences du retrait-gonflement des sols argileux sur ce type de structure est ur objet de préoccupation.

Plusieurs modèles élatoplastiques ont été développés pour décrire le comportement des sols non saturés (Alonso et al., 1987 : Karube et al., 1989; Kohgo et ai., 1991 ; Gallipoli et al., 2003 ; Wheeler et al., 2003...). Ces modèles sont capables de reproduire les principaux traits de comportement hydromécanique caractéristiques des sols non saturés. Alonso et al. \{1987, 1990) ont proposé un modèle élatoplastique pour les sols non saturés non gonflants, nommé BBM (Barcelona Basic Modell. Ce modèle est une extension du modèle de Cam-Clay modifié (Roscoe et al., 1968) dans le domaine des succions non nulles. Il considère que l'état des contraintes des sols non saturés est défini par deux variables indépendantes : la contrainte moyenne nette $\left(\mathrm{p}^{*}\right)$ égale à la différence entre la contrainte totale moyenne $\left(\mathrm{p}=\left(\sigma_{1}+\sigma_{2}+\sigma_{3}\right) / 3\right)$ et la pression de l'air ( $\left.u_{1}\right)$, et la succion (s) définie comme la différence entre la pression de l'air et la pression de l'eau (u, ). Le modèle $B B M$ est capable de décrire correctement : (i) l'effondrement ou le gorflement en fonction de la charge appliquée lors de l'humidilication : (iil l'augmentation de la pression de préconsolidation apparente et de la cohésion du sol avec la succion; (iii) la diminution de la compressibilité avec la succion: (iv) les différentes irréversibilités rencontrées le long des chemins où la succion et la contrainte varient simuitanément, et (v) l'indépendance du chemin de contratinte pour une variation monotone du degré de saturation.

En revanche, le modèle BBM ne tient pas compte de quelques aspects particuliers observés dans des sols gonflants comme : (i) l'apparition du gonflement irréversible lors de l'humidification du sol ; (ii) l'influence de l'étal initial et du chemin de contrainte suivi su' le gonflement et les pressions de gonflement (Brackley, 1973 ; Justo et al., 1984) ; (iii) l'accumulation des déformations de compression ou d'extension au cours des cycles hydriques (Pousada, 1984; Dif et Blumel, 1991), et (iv) la dépendance de la réponse du sol du chemin de contrainte suivi méme tors d'une variation monotone croissante du degré de saturation (Cujsinier, 2002).

Les modèles de comportement volumique des sols gonflants sont actuellement assez peu répandus du fait de leur complexité et de la difficulté des etudes expérimentales couplées tenant compte des effets de changement de succion et de contrainte. Le conportement des sols gonflants a été souvent décrit par des lois relativement simples et empiriques, qui relient la réponse du sol aux changements de la succion et aux contraintes appliquées. Il y a relativement peu de modèles qui intègrent tous Ies aspects principaux du comportement hydromécanique couplé de ces sols dans un cadre unifié. Robinet et al. (1998, 1999) et Baudet et Stallebrass (2004) ont proposé des modèles pour Ies sols gonflants saturés. Cui et al. (2002) ont présenté un modèle élastique non linéaire permettant de décrìre le comportement des sols gonflants non saturés mais fortement compactés. Le modèle proposé par Gens et Alonso (1992) et Alonso ef al. (1999) (BExM ; Barcelona Expansive Model) peut être mentionné comme un modèle de référence pour décrire le comportement des sols gonflants non saturés. Ce modèle a été implanté dans Code_Bright (Mrad, 2005), un code de calcul aux éléments finis développé par le département de géotechnique et geosciences de l'université polytechnique de Catalogne (Espagne) depuis 1996, pour l'analyse des problèmes couplés themo-hydro-mécaniques dans des géomatériaux (Olivella et al., 1996).

Cet article présente une application du modèle $B E x M$ au problème de retrait-gonflement des sols d'assise des fondations superficielles. Dans cette application, l'influence du processus de la saturation et de la désaturation sur le comportement hydromécanique d'un sol gonflant chargé par une semelle filante est étudiée. Une étude de l'influence d'une géomembrane posée dans le sol afin de retarder les transferts hydriques et les phénomènes de retrait-gonflement est également présentée.

\section{2}

\section{Description du modèle $B E X M$}

Le modèle BExM considère une structure à deux échelles pour les sols gonflants, la microstructure qui correspond aux minéraux actifs de l'argile constituant les agrégats, et la macrostructure qui tient compte du reste de la structure du sol et de l'arrangement des agrégats. La microstructure est supposée saturée et présente un comportement réversible indépendant du comportement de la macrostructure, tandis que la macrostructure peut voir son degré de saturation varier. La microstructure est couplée à la macrostructure; ce couplage se traduit par la possibilité d'apparition de déformations plastiques macrostructurales à partir des déformations microstructurales élastiques. 
Le comportement de la macrostructure est défini par la courbe LC (Loading Collapse) qui traduit l'augmentation de la pression de préconsolidation apparente avec la succion (Figure 1a). Le comportement de la microstructure est considéré toujours réversible et ne dépend que de la contrainte effective classique $\left[\mathrm{p}^{\prime}=\left[\mathrm{p}^{*}+\mathrm{s}^{\prime}\right)\right]$. Ceci permet de définir dans le plan $\left(\mathrm{p}^{*}-\mathrm{s}\right)$ une ligne dite $N L$ (Neutral Line) orientée à $45^{\circ}$ par rapport aux axes et qui sêpare la zone du gonflement microstructural de la zone de retrajt microstmictural (Figure 1a). Le couplage entre la microstructure et la macrostructure permet de définir de part et d"autre de la ligne neutre, deux nouvelles lignes notées SI (Suction Increase) et SD (Suction Decrease), qui délimitent la zone des déformations élastiques de la macrostructure en réponse à une solicitation hydrigue.

Ce modèle a été élaboré pour les états de contrainte isotrope et triaxial. Sous chargement isotrope, il utilise deux variables indépendantes : la contrainte moyenne nette $\left(\mathrm{p}^{*}\right)$ et la succion (s). Dans le cas triaxial, une troisieme variable utilisée est la contrainte déviatorique q. La surface de charge dans ce cas est tridimensionnelle (Fig. 1b). Alonso ef al. (1999) supposent que pour des valeurs constantes de la succion, elle se réduit à une ellipse (équation 1).

$$
\mathrm{q}^{3}-\mathrm{M}^{2}\left(\mathrm{p}+\mathrm{p}_{0}\right)\left(\mathrm{p}_{0}(\mathrm{~s})-\mathrm{p}\right)=0
$$

où $M$ est la pente de la courbe d'état critique $s u p-$ posée indépendante de la succion et $p_{\mathrm{s}}=k s$, avec $k$, un paramètre décrivant l'augmentation de la cohésion avec la succion et $p_{o}$ la pression de préconsolidation apparente pour une succion données:

$\frac{p_{0}}{p_{c}}=\left|\frac{p_{0}}{p_{c}}\right|^{\frac{\lambda(0)-x}{\lambda(s)-x}}$ avec $\lambda(s)=\lambda(0)|r+(1-r) \exp (-\beta s)|$

où $p_{0}^{*}$ est la pression de prếconsolidation sous une succion nulle, $p_{c}$ est une pression de référence, kest le coefficient de compressibilité élastique, $\lambda(s)$ est le coefficient de compressibilíté plastique à succion constante, $r$ est un paramètre lié à la rigidité du sol et $\beta$ un paramètre contrôlant le taux d'augmentation de la rigidité avec la succion. Les surfaces de charge SI et SD sont contrôlées par les deux variables d"écrouissage $s_{h}$ et $s_{0}$.

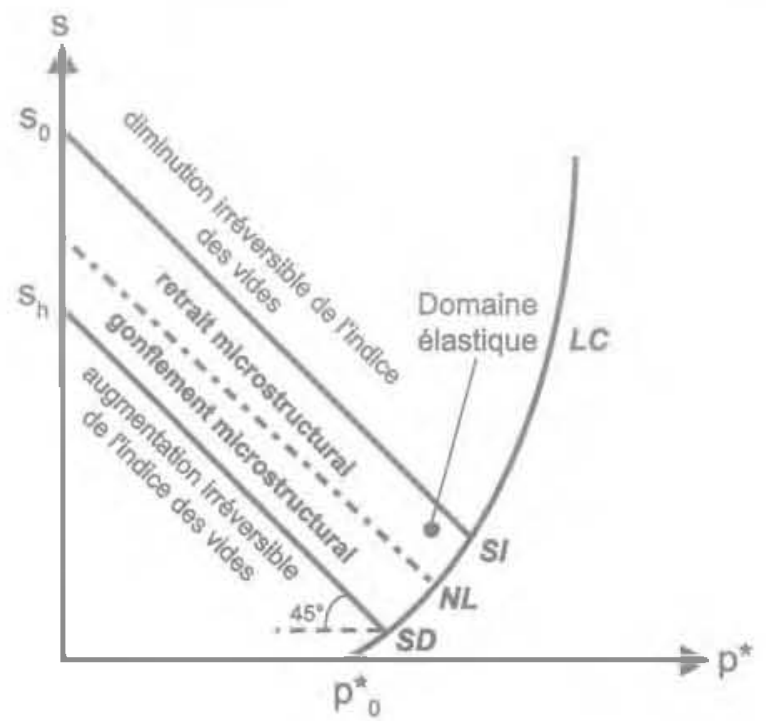

(a)
Les lois d'écrouissage des surfaces de charge SI, SD et LC sont données par :

$$
\begin{aligned}
& d s_{0}=\frac{\left(1+e_{m}\right)\left(p^{*}+s\right)}{k_{m}} \frac{\left(d \varepsilon_{i s 1}^{*}+d \varepsilon_{i s D}^{i}\right)}{f}=d s_{\hbar} \\
& \frac{\mathrm{d} p_{i}^{*}}{p_{i}^{*}}=\frac{\left(1+e_{M}\right)}{\lambda(0)-k}\left(d \varepsilon_{V S I}^{i}+d \varepsilon_{v S D}^{*}+d \varepsilon_{V L C}^{p}\right)
\end{aligned}
$$

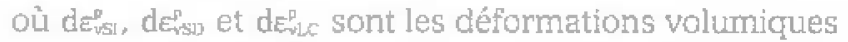
plastiques dues à l'activation respectivement de SI, $S D$ et $L C$ : $\mathrm{e}_{\mathrm{m}}$ et $\mathrm{e}_{\mathrm{M}}$ sont respectivement les indices des vides microstructural et macrosturctural, $\mathbf{x}_{\mathrm{m}}$ est l'indice de compressibilité de la microstructure et $f$ une fonction du couplage micro-macrostructurale (Alonso et al., 1999 ; Alonso et al., 2001 et Iloret et al., 2003).

\section{3}

\section{Implantation et validation}

Le modèle BExM a été implanté dans le code de calcul aux éléments finis Code_Bright (Mrad, 2005). Un algarithme explicite raffiné combiné avec une technique de progression automatique avec contrôle des erreurs a été utilisé pour l'intégration numérique de la relation élastoplastique contrainte-déformation. Cet algorithme est inspiré de celui proposé par Sloan (1987), avec l'extension aux conditions non saturées. Le modèle implanté a été vérifié à l'aide de la simulation des essais cedométriques à succion contrôlée réalisés par Cuisinłer (2002) sur un mélange compacté de $60 \%$ de bentonite et de $40 \%$ de limon, par Lloret et a]. (2003) sur une bentonite contenant plus de $90 \%$ de montmorilionite, et par Romero (1999) sur I'argile de Boom. Ces essais comprennent une combinaison de chemins de chargement à succion constante et de chemins de séchage/humidification a charge constante. Les comparaisons entre les simulations numériques et les mesures expérimentales ont montré que le modèle numérique est en mesure de reproduire quartitativement de façon acceptable les phénomènes caractéristiques du comportement des sols gonflants non saturés

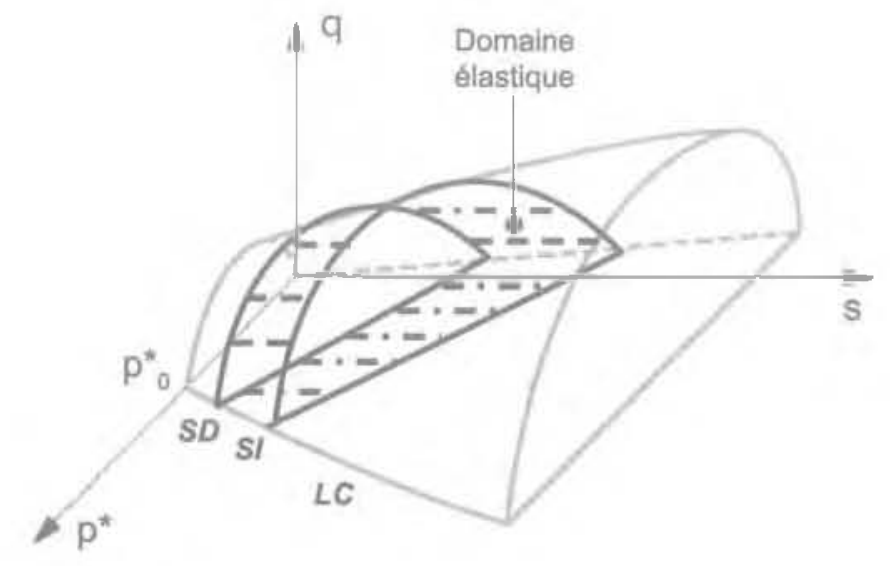

(b)

Fg. 9 Surfaces de charges dans le modèle BExM : (a) dans te plan [p*,s] ; (b) dans l'espace [p*,q,s] (Alonso ef al., 1999).

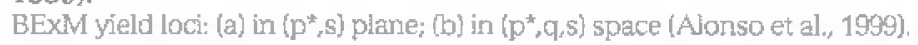


sur des chemins cedométriques (Mrad, 2005; Mrad et al., 2005, 2006).

\section{4}

\section{Application du BEXM à un sol gon- flant chargé}

Dans ce qui suit, sont étudiés les déplacements verticaux d'un sol gonflant chargé par une semelle filante en béton et exposé à des variations de conditions climatiques. Le choix de la modélisation d'un tel type de fondation a été basé sur l'analyse statistique d'un grand nombre de sinistres dus au phénorè̃e de retrait-gonflement réalisée par İ CEBTP-Solen dans le cadre du projet Retrait-Gonflement-RGC\&U (Vincent et al., 2006). Cette analyse montre que les principaux bâtis gravement endommagés par les phénomènes de retrait-gonflement sont des maisons individuelles, dont les fondations sont dars la grande majorité des cas $(95 \%$ ) des semelles filantes. Dans $87 \%$ des cas de sinistre, le niveau d"assise des fondations est inférieur à 1,20 m de profondeur par rapport à la surface.

Les modélisations numériques ont été réalisées en utilisant le Code_Bright où le modèle BExM, servant à décrire le comportement du sol gonflant, a été implémenté.

\section{1}

\section{Caractéristiques de la fondation}

La fondation est posé̉ à $60 \mathrm{~cm}$ de profondeur dans le sol gonflant (Fig. 2). Le béton de la semelle suit un comportement de type élastique linéaire. Une contraînte verticale de $300 \mathrm{kPa}$ est appliquée à sa base. Ses propriétés mécaniques et hydrauliques sont rassemblées dans le tableau I (Burlion et al., 2005).

\section{2}

\section{Caractéristiques du massif de sol}

La fondation superficielle repose sur une couche limitée à $1,6 \mathrm{~m}$ d'épaisseur d'argile gonflante homogène (Fig. 2) et un sol non gonflant qui a un comportement de type élastique linéaire. Cette argile a les caractêristiques d'un mélange limon-bentonite compacté (Cuisinier. 2002 ; Cuisinier et Masrouri, 2004). Son comportement élastoplastique est représenté par le modèle BExM dont les paramètres ont été déterminés à partir d'essais réall sés au laboratore (Mrad, 2005) (tableaux I et II).

Ce sol argileux possède une perméabilité à la saturation de $5 \times 10^{-9} \mathrm{~m} / \mathrm{s}$. Les paramètres de la courbe de rétention ont êté déterminés par ajustement de la courbe expérimentale de Cuisinier (2002) par le modèle de van Genuchten (1980) (Fig. 3 et tableau I).

La prise en compte de la fouille creusée pour réaliser la fondation a été introduite dans le modèle par la considération d'une zone à plus grande perméabilité, située au-dessus de la fondation: "remblai . Le comportement hydrique de ce remblai plus perméable que le sol environnant influence les résultats de la modélisation (Fig. 3 et tableau I). Son comportement mécanique est supposé régi par le modêle BExM dont les paramètres sont identiques à ceux de l'argile gonflante (tableau I)).

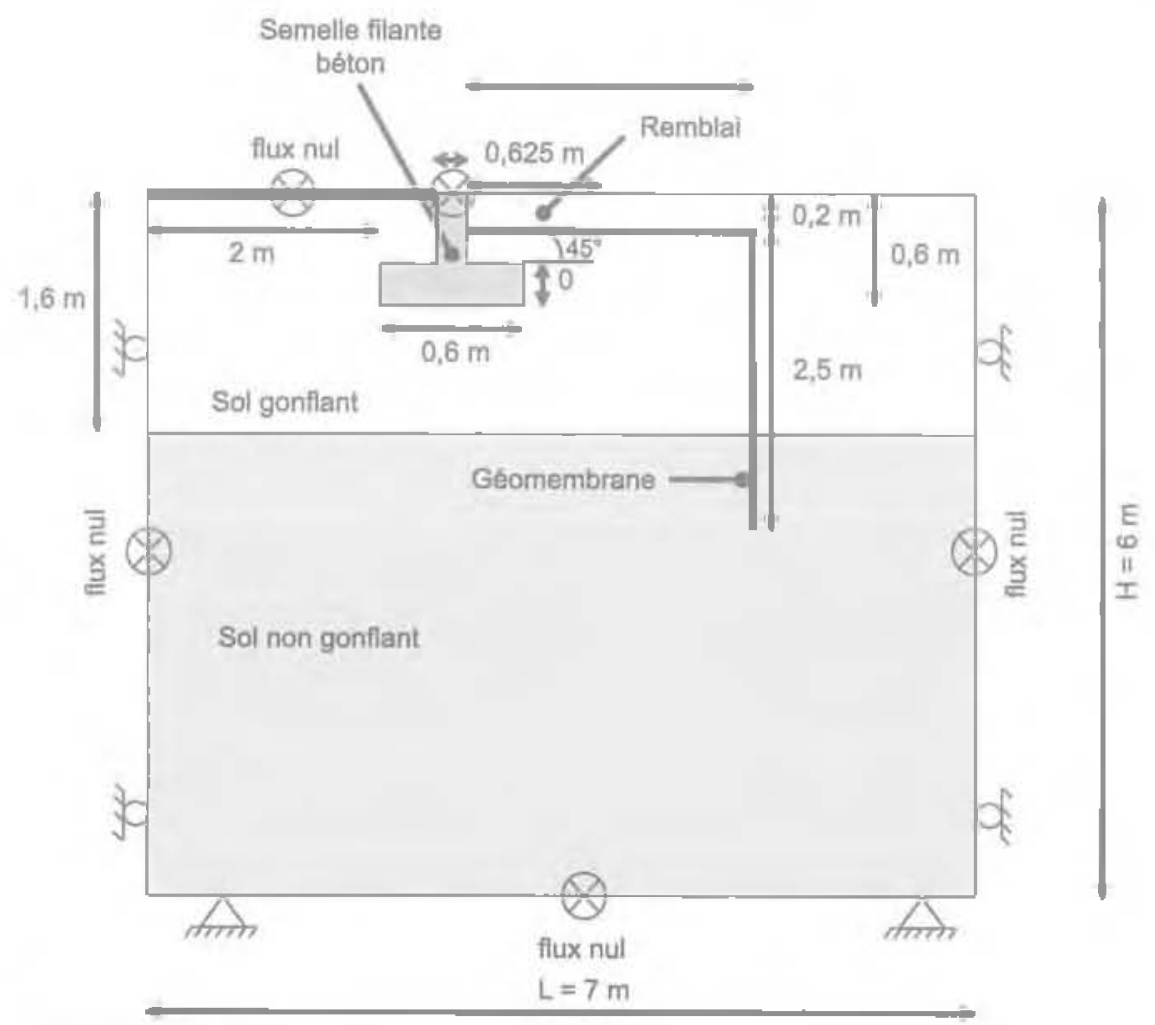

Fig. 2 Géométrie et conditions aux limites du modèle. Geometry and bolindary conditions of the model. 
zalsau II Paramètreg caractérisant les matériaux du modêle.

Parameters characterizing materials of the model.

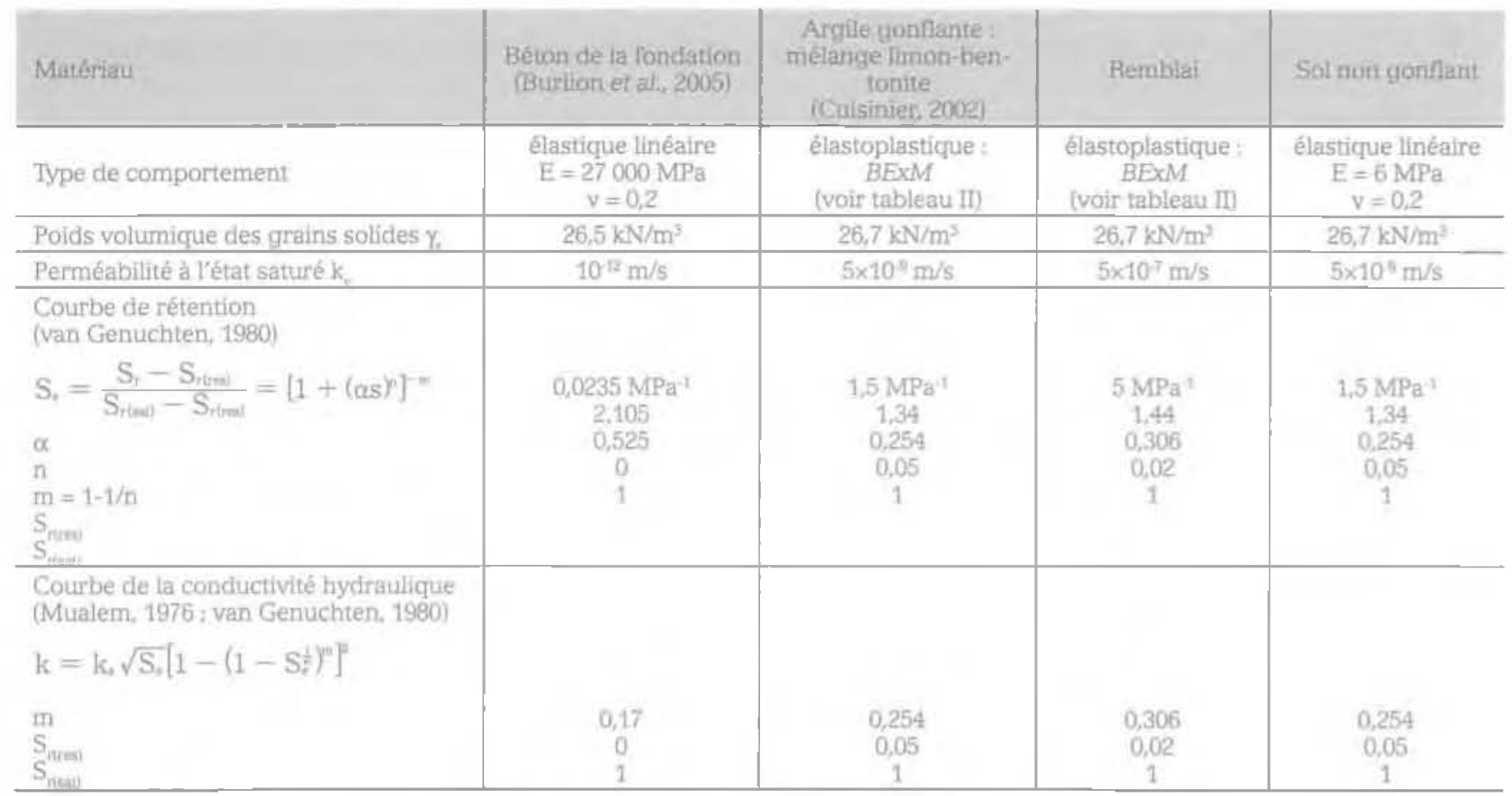

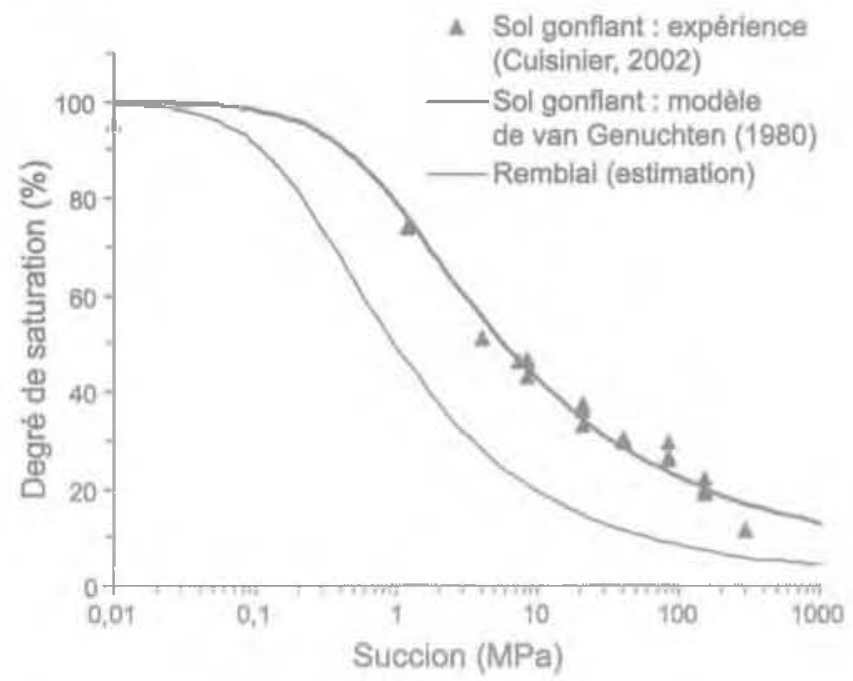

Fls. 3 Courbes de rétention du sol argileux et du remblai.

Soil water relention curves of the sill-bentonite mixture and the backfill.

\section{3}

\section{Influence de la mise en place d'une géomembrane}

Pour limiter l'évaporation, il est recommandé de mettre en place une géomembrane en périphérie de la fondation (Vincent et al., 2006). Une deuxième modélisation a donc été effectuée en considérant une couche de gếomemliane posée dans le sol à $20 \mathrm{~cm}$ de la surface, qui s'étend à une distance de 2,5 m et qui pénètre dans le sol jusqu'à $2,5 \mathrm{~m}$ de profondeur. Cette géomembrane a une épaisseur de $4 \mathrm{~mm}$ et un coefficient de perméabilité de $10^{14} \mathrm{~m} / \mathrm{s}$ (Bordes, 1995).

\section{4}

\section{Le modèle et les conditions initiales et aux limites}

La fondation est modélisée en déformation plane. L'imperméabilisation du côtế intérieur du bâtiment dû̀ à l'existence du plancher a été prise en compte. Le massif de sol a été discrétisé dans son intégralité par des éléments finis rectangulaires isoparamétriques à 4 noeuds. Le mêrne type d'élément a été adopté pour le maillage de la fondation, afin d'assurer un assemblage correct. Le maillage est constitué en totalité de 1436 éléments et 1508 nouds.

Les conditions initiales du modèle sont définies dans le tableau III et les conditions aux limites sont représentées sur la figure 2 .

\section{5}

\section{Phasage de calcul}

Pour cette étude, les conditions climatiques correspondant aux données météorologiques issues des mesures effectuées entre 1998 et 1999 sur un terrain situé à Rambouillet, France (Beauchamp, 2006) ont été considérées (tableau IV). Elles montrent que la période entre novembre et févier est caractérisée par de fortes précipitations. Tandis que, les résultats du bilan hydrique pour les autres mois correspondent à une période de sécheresse.

Pour le calcul trois phases consécutives ont été considérées. Dans chaque phase hydrique, une condition simulant une précipitation ou une évaporation a été imposée à la surface du so] [tableau IV] :

- phase I (novembre-février), Pendant les quatre mois de précipitations, les valeurs de flux d'eau infiltré dépassent la perméabilité à la saturation, la capacité d'infiltration du sol est alors atteinte : par conséquent, 
zantinu II Paramètres du modèle BExM pour Ie mélange bentonite-limon et pour le remblai. Parameters of the BExM model for the silt-bentonite mixture and the backfill.

Paramètres définissant la loi de comportement macrostructural

\begin{tabular}{c|c}
$\kappa$ & 0,02 \\
$\lambda(0)$ & 0,315 \\
$r$ & 0,2777 \\
$\beta$ & $8,054 \times 10^{-5} \mathrm{MPa}^{-1}$ \\
$\kappa_{s}$ & 0,015 \\
$p_{c}$ & $0,609 \mathrm{MPa}$ \\
\hline
\end{tabular}

\begin{tabular}{c|c}
$\mathrm{P}_{0}^{*}$ & $0,844 \mathrm{MPa}$ \\
$\mathrm{s}_{0}$ & $30 \mathrm{MPa}$ \\
$\mathrm{s}_{\mathrm{h}}$ & $14 \mathrm{MPa}$ \\
$\mathrm{k}$ & 0,09 \\
$\mathrm{M}$ & 1,24
\end{tabular}

Paramètres définissant la loi du comportement microstructural

\begin{tabular}{l|l|l|l}
$\kappa_{\mathrm{m}}$ & 0,011 & $e_{m}$ & 0,25
\end{tabular}

Fonctions de couplage micro-macrostructurale

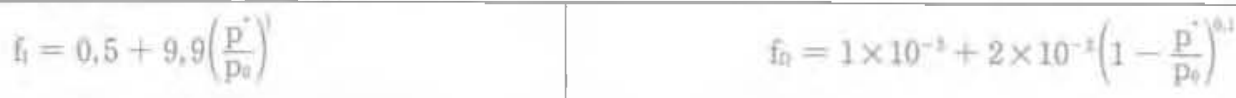

pour" simuler la précipitation une succion nulle à la surface de sol a été imposée;

- phase IJ (mars). La modélisation de l'évaporation a étế réalisée en imposant un flux d'eau négatifs de $1,37 \times 10^{-6} \mathrm{~m} / \mathrm{s}$ à la surface du sol ; à la fin de cette phase, une succion de $20 \mathrm{MPa}$ est obtenue à la surface ;

- phase III (avril-octobre). Dans cette phase, l'imposition du flux en surface aboutit à des grandes valeurs de succion, ce qui entraîne des problèmes numériques de convergence. Il a donc été décidé d’appliquer la succion de $20 \mathrm{MPa}$ obtenue dans la phase précédente à la surface de sol. Par ailleurs, cette valeur correspond à la succion au-delà de laquelle la désaturation du sol engendre une faible variation d"indice des vides (Cuisinier, 2002).

\section{6}

\section{Résultats}

Dans ce paragraphe, sont présentés les principaux résultats des modélisations et les comparaisons des deux cas avec et sarıs géomembrane.

\section{acia \\ Succions et degrés de saturation}

Les figures $4 a$ et $4 b$ présentent les courbes d'évolution temporelle de la succion et du degré de saturation. Elles permettent de visualiser les phénomènes de transfert hydrique pour differents points (A à D) dans le sol situés sous la base de la fondation à une profondeur de

zantżu ill Conditions initiales du modêle (succion initiale = $20 \mathrm{MPa}$ ), Initial Conditions of the model.

\begin{tabular}{|c|c|c|c|c|}
\hline Matériau & Béton te la londation & $\begin{array}{c}\text { Argtle gonflante } \\
\text { (mélange } \\
\text { Imon-bentonite) }\end{array}$ & Remblai & $\begin{array}{l}\text { Sol } \\
\text { non gonflant }\end{array}$ \\
\hline Porosité n & 0,16 & 0.524 & 0.6 & 0,524 \\
\hline Indice des vides e & 0.19 & 1.1 & 1,5 & 1,1 \\
\hline Poids volumique sec $\gamma_{*}\left(\mathrm{kN} / \mathrm{m}^{3}\right)$ & 22,3 & 12,7 & 10,7 & 12,7 \\
\hline
\end{tabular}

uabiau Donnés météorologiques à Rambouillet, France (Beauchamp, 2006) et phasage de calcul. Meteorological data in Rambouillet. France (Beauchamp, 2006) and calculation phases.

\begin{tabular}{|c|c|c|c|c|c|}
\hline Mois & $\begin{array}{c}\mathrm{P} \\
\text { (min/mois) }\end{array}$ & $\begin{array}{c}\text { ETP } \\
\text { (mm/ntois) }\end{array}$ & $\begin{array}{l}\mathrm{P}-\mathrm{ETP} \\
\text { [mm/mois] }\end{array}$ & $\begin{array}{c}P-E T P \\
(m / s)\end{array}$ & $\begin{array}{c}\text { Conditions aux limites appli- } \\
\text { quêes en surface }\end{array}$ \\
\hline Novermbre & 53,4 & 9,2 & 44,2 & $1,71 \times 10^{9}$ & \multirow{4}{*}{$\begin{array}{l}\text { Phase I : } \\
\text { Succion }=0\end{array}$} \\
\hline Décembre & 70,3 & 17,1 & 53,2 & $1.99 \times 10^{\theta}$ & \\
\hline Janvier & 41,4 & 16,2 & 25,2 & $9,41 \times 10^{9}$ & \\
\hline Février & 86,5 & 35,1 & 51,4 & $2,12 \times 10^{\mathrm{B}}$ & \\
\hline Mars & $\$ 4,1$ & 50,9 & $-36,8$ & $-1,37 \times 10^{-8}$ & $\begin{array}{c}\text { Phase II : } \\
\text { Flux d'eau évaporé } \\
=-1,37 \times 10^{8} \mathrm{~m} / \mathrm{s}\end{array}$ \\
\hline Avril & 64,3 & 80,9 & $-16,6$ & $-6,40 \times 10^{9}$ & \multirow{6}{*}{$\begin{array}{l}\text { Phase } 1 \mathrm{II} \\
\text { Succion }=20 \mathrm{MPa}\end{array}$} \\
\hline Mai & 9.1 & 120,6 & $-115,5$ & $-4,31 \times 10^{8}$ & \\
\hline Juin & 56,2 & 104,7 & $-48,5$ & $-1.87 \times 10^{-18}$ & \\
\hline Juillet & 39,4 & 143,2 & $-103,8$ & $-3,88 \times 10^{-8}$ & \\
\hline Août. & 11,8 & 118,7 & $-106,9$ & $-3,99 \times 10^{-8}$ & \\
\hline Octobre & 28,6 & 40,0 & $-11,4$ & $-4,26 \times 10^{\circ}$ & \\
\hline
\end{tabular}


$65 \mathrm{~cm}$, dans les cas avec (a) et sans (s) géomembrane. L'allure générale de ]'évolution du degré de saturation. est logiquement semblable à celle de la succion.

Dans le cas sans géomembrane, la phase d'humidjfication (phase I) entraine une augmentation rapide des valeurs du degré de saturation qui décroissent progressivement avec le temps pendant les phases de séchage (phases II et II). Comme Ie plancher du bâtiment et la semelle en béton constituent des écrans contre l'écoulement, le sol sous-jacent s'humidifie moins vite que les autres points situés au même niveau mais à l'extérieur de la fondation. A la fin de la phase d'humidification, les valeurs de la succion sous le plancher (point A) sont supérieures à celles des autres points situés à l'extérieur de la fondation, le transfert hydrique vers le point A continue alors pendant les phases de séchage.

La mise en place de la géomembrane limite de manière considérable le phénomène d"humidification-séchage, plus particulièrement aux points A et C. Contrairement à ce que l'on a observé dans le cas sans géomembrane, le sol sous le centre de la fondation (point B) s'humidifie plus vite que le point $C$ situé sous la géomembrane. Le transfert de l'eau pour le point B continue pendant le débul des phases de séchage et ce jusqu'à 180 jours. Le comportement hydrique du point D, à l'extérieur de la géomembrane, est similaire dans les deux cas, avec et sans géomembrane.

\section{6 .8}

\section{Déplacements verticaux}

La figure 5 a présente la comparaison de l'évolution temporelle du déplacement vertical qui est considérablement influencée par la présence de la géomembrane. Le chargement mécanique a engendré un déplacement maximum de $35 \mathrm{~mm}$ pour le point $\mathrm{B}$ situé sous la semelle. Pendant la phase d'humidification. le sol gonfle progressivement avec le temps. A la Pin de cette phase, la mise en place de la gêomembrane réduit les gonflements du point $\mathrm{D}$ de $72,6 \mathrm{~mm}$ à $54 \mathrm{~mm}$, du point $\mathrm{C}$ de $51 \mathrm{~mm}$ à $27,4 \mathrm{~mm}$ et du point $\mathrm{B}$ de $45 \mathrm{~mm}$ à $8,8 \mathrm{~mm}$. Pour le point situé sous le bâtiment (point A), au lieu de gonflement de $17 \mathrm{~mm}$, la présence de la géomembrane induit un tassement de 22,7 mm. Pour ce même point, le début de la phase d'humidification comporte une période où le sol tasse avant de gonfler.
Ce qui est dû à un gonflement plus important du sol se situant côté extérieur (droite) de la semelle qui engendre une rotation de la fondation rigide. Les phases de séchage produisent, un tassement à l'extrémité du modèle (point D) de $11 \mathrm{~mm}$ dans le cas sans géomembrane et de $6 \mathrm{~mm}$ dans le cas avec géomembrane et un tassement au point $\mathrm{C}$ de l'ordre de $6 \mathrm{~mm}$ dans les deux cas et une stabilisation du gonflement pour les autres points (points A et B). Ces résultats montrent que l'existence de la géomembrane diminue et retarde les variations de volume du massif de sol de manière importante.

\section{6 .3}

\section{Analyse des chemins de contraintes}

La figure 6 montre les chemins de contrainte suivis au cours des phases de calcul dans le plan $\left(p^{\prime \prime}, s\right)$ pour le point $B$ situé sous le centre de la fondation dans le cas du modèle BExM. La phase d'humidification provoque la plastification du sol, après 22 jours de précipitation dans le cas sans géomembrane, et après 88 jours de précipitation dans le cas avec géomembrane. En effet, cette phase d'humidification crée un dépassement de la courbe $S D$ de sa position initiale $S D$ vers sa position finale $S D_{r} \mathrm{Ce}$ déplacement est couplé avec un mouvement de la courbe LC (LC vers LC ) et de la courbe SI (SI, vers SI ). Les chemins de contrainte au cour's des phases de séchage se développent donc dans la zone êlastique. Ceci explíque les faibles valeurs du tassement du sol pendant les phases de séchage pour le modêle BExM (Figure 5a).

\section{6 .4}

\section{Apport du modèle BExM}

Afin de montrer l 'apport du modèle utilisé (BExM), les mêmes calculs ont été effectués en supposant cue le sol de la fondation est élastoplastique et représenté par le modèle $B B M$ pour les sols non saturés (Fig. 5b). La comparaison de ces résultats montre que les déplacements en humidification et en retrait sont sous-estimés dans le cas du modèle BBM qui ne prend pas en compte le gonflement irréversible lors de l'humidification (i.e. surface de charge SD). 


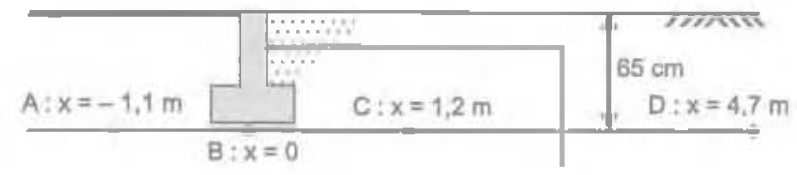

$\because A(s) \multimap B^{\prime}(s) \rightarrow C^{(s)} \leftrightarrow D^{\prime}(s)$
$\because A^{\prime}(a) \multimap B^{\prime}(a) \rightarrow C^{\prime}(a) \rightarrow D^{\prime}(a)$

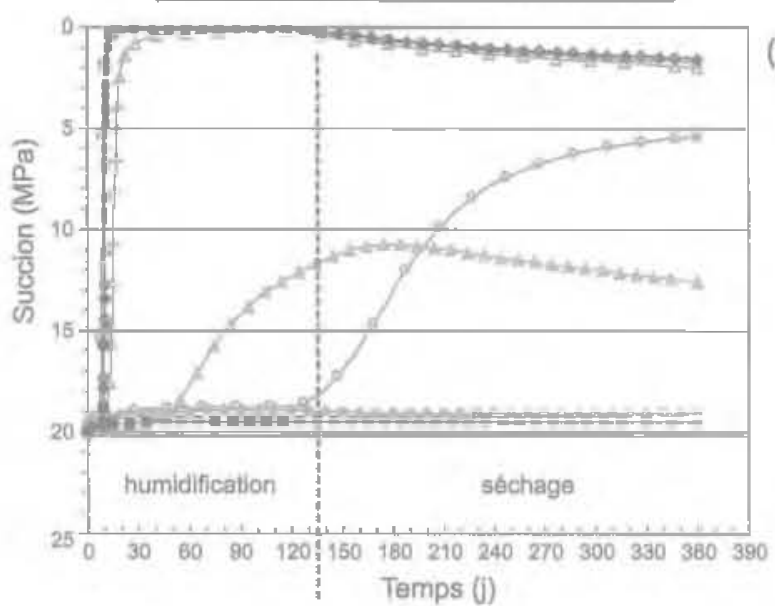

(a)

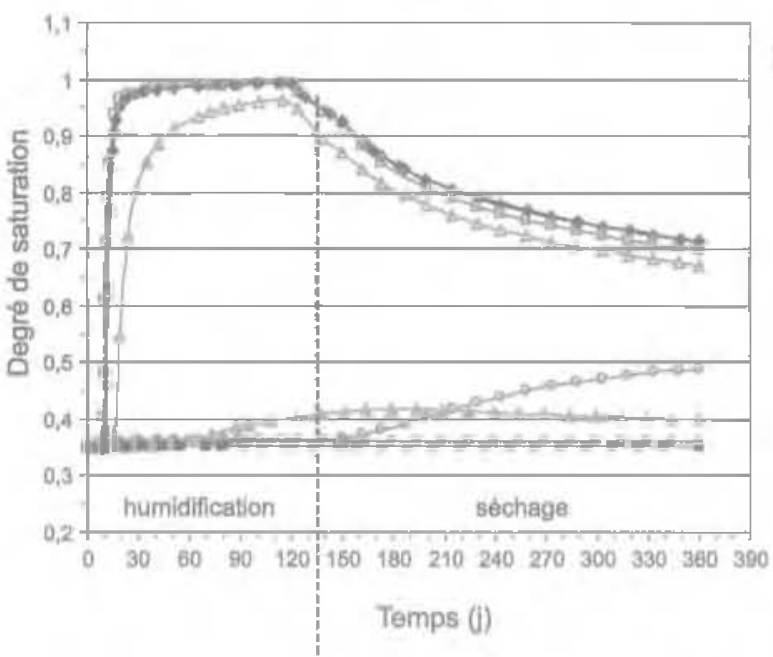

(b)

FG. 4 Comparaison de l'évolution temporelle ; a) de la succion ; et b) du degré de saturation, avec (a) et sans (s) géomenbrane.

a) Suction and (b) degree of saturation. versus tine in the soil, with (a) and without (s) geomembrane protection.
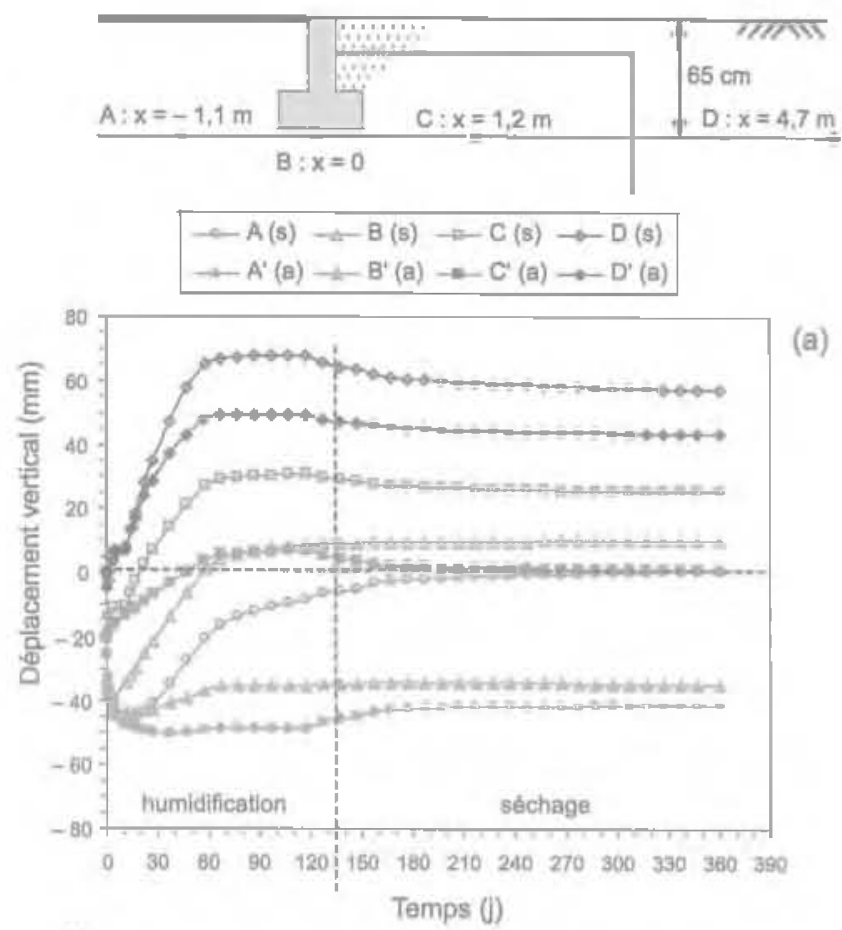

(a)

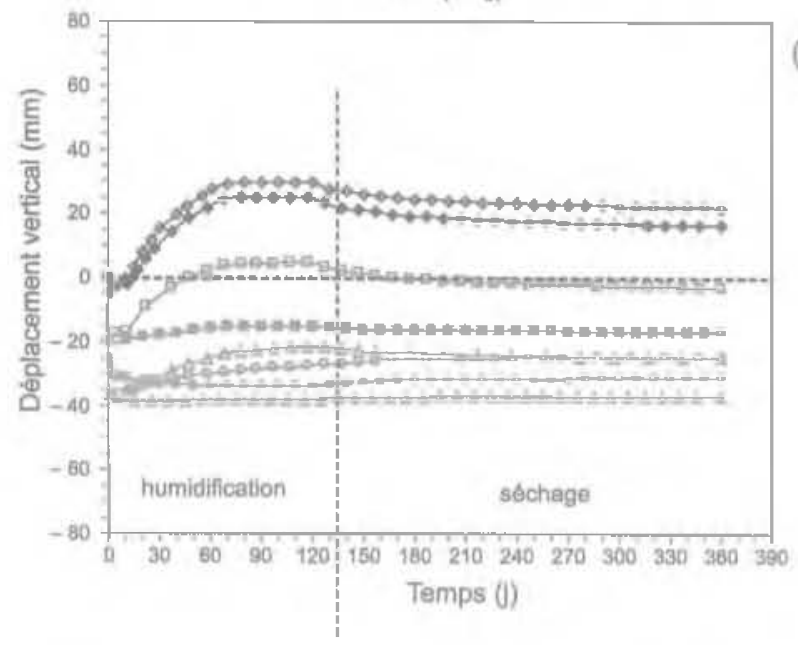

(b)

Fas 5 Comparaison de J'évolution temporelle du déplacement vertical avec (a) et sans (s) géomembrane prédites par les : a) modéle $\mathrm{BExM}$; b) modèle BBM.

Comparison of the vertical displacement with (a) and without (s) geomembrane protection predicted by the: a) BExM model; b) BBM model. 

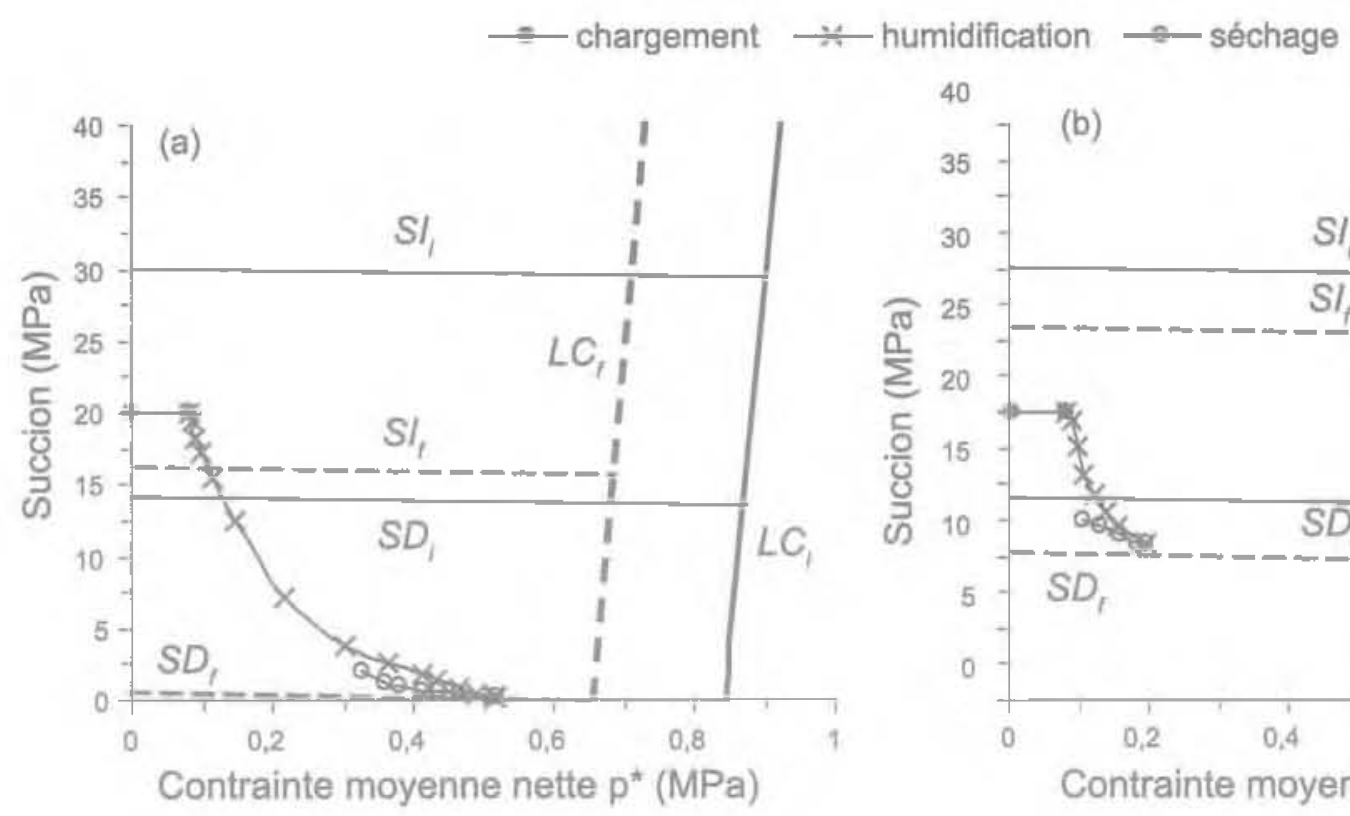

40

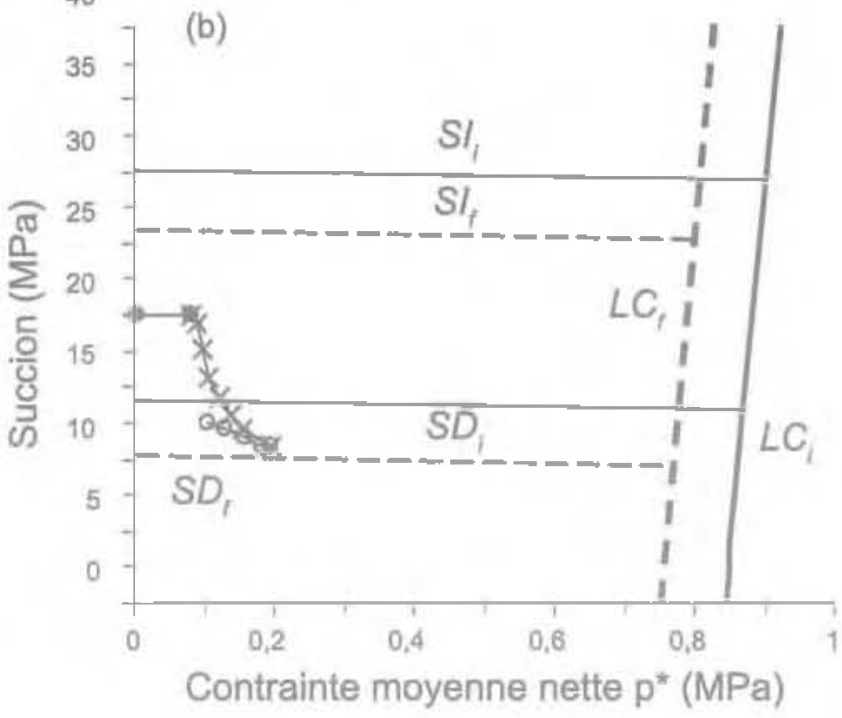

Ma. 6 Chemins de contrainte suivis par le centre de la fondation (point B) au cours des phases de calcul : a) sans géomembrane; ; b) avec géomembrane.

Stress-paths on the soil under the centre of the foundation (point B): a) with geomembrane protection; b) without geomembrane protection.

\section{5}

\section{Conclusion}

Dans cet article, le modèle élastoplastique (BEXM) a été présenté, il permet de décrire la plupart des phénomènes hydromécaniques qui ont été constatés expérimentalement dans le cas des sols gonflants non saturés.

Une application de ce modèle, implanté dans le Code_Bright, à la modélisation du problème de retraitgonflement d'une couche d'argile gonflante chargée par une fondation superficielle a été présentée. Les résultats ont montré que le modèle est capable de décrire qualitativement les déplacements du massif de sol pendant les différentes sollicitations hydriques. Le chargement mécanique engendre un déplacement maximum sous le centre de la fondation. Le gonflement sous ta base de la semelie dü uniquement à la précipitation est plus faible que celui des autres points situés au môme niveau mais à lextérieur de la semelle. car cette derrière constitue elle-même un écran contre l'écoulement dans le cas où les chemins préférentiels d'écoulement le long de la fondation ne sont pas pris en compte.

Les résultats d'une modélisation avec la prise en compte d'une géomembrane posée dans le sol, mon- treat que cet écran limite le gonflement du massif de sol de manière importante et son utilisation pour retarder les transferts hydriçues et les phénomènes de retrait-gonflement est valable.

La comparaison des déplacements verticaux prédits par les deux modèles BEXM et BBM a montré la capacité du premier modèle à prendre en compte l'apparition des gonflements irréversibles lors de l'humidification du sol, qui n'étaient pas prise en compte dans le cadre du modèle $B B M$.

A ce stade, il serait intéressant de disposer des résultats des mesures in situ des déformations volumiques lors des cycles de séchage-humidification dans des sols gonflants, afin de valider les résultats numériques obtenus.

\section{REMERCIEMENTS}

Ces travaux ont été rếalisés dans le cadre du projet de recherche irationa1: " Analyse du Retrait-Gonflement et de ses Incidences sur les Constructions y (ARGIC) financé par l'Agence nationale de la recherche (ANR) - Réseau Génie Civil et Urbain RGC\&U). Les auteurs tiennent à remercier l'ensemble de partenaires de ce projet, tout particulièrement pour la coordination M. Marc Vncent, ingénieur de recherche au BAGM, unité Amêragement et Risques naturels. 
Alonso E.E., Gens A., Hight D.W. - General repart. Special problem soils. Proceedings of the Sth European Conference on Soil Mechanics and Foundation Engineering, Dublin, vol. 3, 1987, p. 10871146.

Alonso E.E., Gens A., Josa A. - A constitutive model for partialiy saturated soils. Gétechnique, vol. 40, n 3. 1990, p. 405430.

Alonso E.E., Vaunat J, Gers A. - Modelling the mechanical behaviour of expensive clays. Engineering Geology, vol. 54, 1999, p. $173-183$.

Alonso E.E., Romero E., Hoffmann C., Garcia-Escudero E. 2001. Expansive bettonite/sand mixtures in cyclic controlled suction drying and wetting. Proceedings of the 6th International Workshop on Key Issues in Waste Isolation Research, Faris, Ecole nationale des ponts et chaussées, 2001, p. 513-54?

Baudet B., Stallebrass - A constitutive model for structured clays. Géotechnique vol. $54, n^{\circ} 4,2004$, p. $269-278$

Beauchamp J. - L'eau et le sol. [En ligne : enregistré le 21 aout 2006] http://www. oleiculteur.com/ L'eau\%20et\%20le\%20sol.htm. 2006.

Bordes J.-L. - Géomembranes : utilisation en gênie civil. Techniques de l'ingénieur, traite construction, article C5430. 1995, $14 \mathrm{p}$.

Brackley I.J.A. - Swell pressure and free swell in compacted clay. Proceedings of the 3rd International Conference on Expansive Soils, Haifa, vol. 1, 1973, p. $169-176$.

Burlion N., Bolurgeois F, Shao J.F. -Effects of desiccation on mechanical behaviour of concrete. Cements \& Concrete Compasites, vol. 27, 2005, p. 367-379.

Chen F.H. - Foundations on expansive soils. Amsterdam, Elsevier. Developments in Geotechnical Engineering, vol. 12, 1975, $280 \mathrm{p}$ -

Cuí Y.J., Yahia-Aissa M., Delage P. - A model for the volume change behavior of heavily compacted swelling clays. Engineering Geology, vol. 64, 2002, p. 233-250.

Cuisinier 0. 2002. Comportement hydromécanicue des sols gonflasts compactés. Thèse de doctorat, INPI, Nancy, $176 \mathrm{p}$.

Cuisinier O, Masrouri F. - Testing the hydromechanical behaviour of a compacted swelling soil. ASTM, Geotechnical Testing Joumal, vol. 27, $\pi^{8}$ 6, 2004, p. 598-606.

Derriche Z., Iguechtal L., Tas M. - Comportement des ouvrages dans les argiles expansives d'In-Aménas. Revue française de géotecnhique, $\mathrm{n}^{\circ}$ 89, 1999 , p. $55-65$.
Dif A.E., Bluemel W.F. 1991. Expansive soils under cyclic drying and wetting. Geotechnical Testing Journal, vol. 14, $n^{\circ} 1$, 1991, p. 96-102.

Gallipoli D., Gens A., Sharma R. , Vaunat J. - An elasto-plastic model for unsaturated soil incorporating the effects of suction and degree of saturation on mechanical behaviour. Géotechnique, vol. $53, n^{\circ} 1,2003$, p. 123-135.

Gens A., Alonso E.E. 1992. A tramework for the behaviour of unsaturated expansive clays. Canadian Geotechnical Journal, wol. 29, 1992, p. 1013-1032.

Hachichi A., Fleureaı J.M. - Caractérsation et stabilisation de cuelques sols gonflants d'Algérie. Revue française de géotechnique, n' ${ }^{\circ}$ 86, 1999, p. 37-51.

Justo J.L., Delga do A. Ruiz J. - The influence of stress-path in collapse-swelling of soils at the laboratory. Proceedings of the Sth International Conference on Expansive Soils, Institution of Engineers of Australia, Adelaide: vol, 1, 1984, p. 6771.

Karube D. \& Kato S. - Yield functions of unsaturated soils. Proceedings of the 12th International Conference on Soil Mechanics and Foundation Engineering. vol. 1, 1989, p. 615-618.

Kohgo Y., Nakano M., Miyazaki T. - Elástoplastic constitutive modelling for unsaturated soils. Computer Methods and Advances in Geomechanics, Balkeme, 1891, p. 631-636.

Lloret A. Vilar M.V., Sänchez M. Gens A. Pintado X. Alonso E.E. - Mechanical behaviour of heavily compacted bentonite under high suction changes. Gétechifique, vol. $53, \pi^{\circ} 1,2003$, p. $27-40$.

Mrad M. 2005. Modélisation du comportement hydromécanique des sols gonflants non saturés. Thèse de doctorat, [NPL. Nancy, $244 \mathrm{p}$

Mrad M., Cuisinier O., Abdallah A, Masrouri F. - Modélisation du comportement hydromécanique des sols gonflants non satures sous fortes succions. Comptes rendus du XVIe Congrès interrational de mécanique des sols et de la géotechnique. Osaka, Japon, 2005, p. 551-554,

Mrad M., Abdallah A., Masrouri E., Vaunat J. - Finite-Element implementation of $B$ ExM elastoplastic model for swelling unsaturated soils. Proceedings of the 4 th International Conference on Unseiturated Soils, Arizona, USA, 2006

Mualen Y. - A new model for predicting the hydraulic conductivity of unsaturated porous media. Water Resources Res., vol. 12,1976 , p. 513-522.

Qlivella S., Gens A., Carrera J., Atonso E.E. - Numerical formulation for a simulator
(Code_Bright) for the coupled analysis of saline media. Engmeering Computations, vol. $13, n^{\circ} 7,1996$, p. $87-112$.

Philipponat G. - Retrait-gonflement des argiles, proposition de méthodologie. Revue française de géotechnique, $n^{3} 57$, 1991 , D. 5-22.

Pousada E. 1984. Deformabilidad de arcillas expansivas bajo succion controlada. Thèse de doctorat, Universidad Politecnjca de Madrid 1984, Espagne, 1984.

Robinet J. C., Pothier C., Jullien A., Plas F 1998. Evaluation of a constltutive mode for expansive and nor expansive clays. Proceedings of the 5th international Workshop on Key Issues in Waste Isolaton Research, Barcelona, Spain. 1998

Robinet J. C., Pakzad M., Jullien A., Plas F 1999. A ceneral modelling of expansive and non-expansive clays. Internationa! Jouna! For Numerical and Analytica! Methods in Geomechanics, vol. 23, 1999, p. $1319-1335$.

Romero E. - Characterization and thermohydro-mechanical behaviour of unsaturated boom clay: an experimental study PhD thesis, Technical University of Catalonia, Spain, 1999, 405 p.

Roscoe K.H., Burland J.B. - On the generalized stress-strain behaviour of the wet clay. Engineering Plasticity, Heyman, J, Leckie, FA. (Eds), Cambridge University Press, Cambridge 1968, p. 535-609.

Sloan S.W. - Subtepping schemes for the numerical integration of elastoplastic stress-strain relations. International Jownal for Numerical Methods in Engineering, vol. 24, 1987, p. $893-811$

Vandangeon P. - Exemples de sinistres en région parisienne. Revue française de géotechnique, $n^{\circ} 58,1992$, p. 7-14

Vincent M., Bouchut J., Fleureau J.M., Masroury F., Oppenheirn E., Heck J.V, Ruaux N., Le Roy S., Dubus I., Surdyk N. - Étude des mécarismes de déclenchement du phénomène de retraltgonflement des sols argileux et de ses interactions avec le bâti. Rapport final du projet AGC\&U, BEGM/RP-54862-FR, octobre, 2006.

van Geruchten M.TH. - A closed-form equation for predicting the hydratHc conductivity of ursaturated soils. Soil Science Society American Journal, vol. 44, 1980, p. 892 - 898.

Wheeler S.J. Sharma R.S., Buisson M.S.R. 2003. Coupling of hydraulic hysteresis and stress-strain behaviour in unsattrated soils. Géotechnique, vol. $53, \Omega^{\circ} 1$, 2003, p. $4 t-54$. 